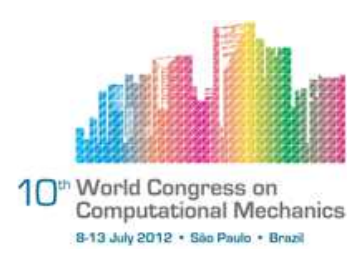

\title{
A Numerical Simulation for Aberrant Crypt Foci
}

\author{
G. Romanazzi ${ }^{1 *}$, I.N. Figueiredo ${ }^{1}$, C. Leal ${ }^{1}$ \\ ${ }^{1}$ Centro de Matemática, Universidade de Coimbra \\ *Corresponding author (roman@mat.uc.pt)
}

\begin{abstract}
.
It is generally accepted that colorectal cancer (CRC) occurs as a consequence of several genetic mutations in the colonic mucosa. The detection and assessment of aberrant crypt foci $(A C F)$, which are clusters of abnormal colonic crypts and believed to be the precursors of $C R C$, is therefore of crucial importance.

This paper models and describes the dynamics of abnormal cells in the colon epithelium, with the goal of simulating the appearance and evolution of ACF.

The colon is represented by a rectangular periodic domain, consisting of small rectangles containing at their centers small circles (these symbolize the crypts) and the exterior of the circles represents the intercryptal regions. The dynamics of the abnormal cells is modeled by a convection-diffusion system (where the convective velocity is defined by a Darcy's law), inside the crypt, and by a pure diffusive model in the intercryptal region. Moreover this model is coupled with a level set equation for tracking the boundary of the abnormal cell set.

Numerical simulations are reported and discussed for the particular case of a periodic domain consisting only of two rectangles.
\end{abstract}

Keywords: Convective-Diffusive Model; Level Set Method; Colonic Crypts.

\section{Introduction}

Colorectal Cancer (CRC) is one of the most frequent types of malignant tumors in the world [1]. Unlike most other kind of cancer, it is possible to prevent CRC. This is due to the long period of time elapsed between the appearance of an adenoma and the eclosion of the carcinoma. This processus is called adenoma-carcinoma sequence. Earliest events in the process development of adenomas are the ACF that can be detected by clinical observation.

There are no certainties about the molecular pathology of the ACF morphogenesis, two different theories have been proposed: the top-down [8] and the bottom-up [7] theories. In top-down morphogenesis, the initial mutation appears in a cell in the intercryptal region, that expands laterally and enters and fills the adjacent normal crypts. In bottom-up morphogenesis, the first abnormal cell occurs at the base of the crypt, where there exists a strong proliferative activity that promotes its increase in number, until they eventually fill the entire crypt. Different experimental results support each hypothesis. There is even strong evidence that 
both situations can occur during ACF morphogenesis, and that the processes are not mutually exclusive.

The goal of this work is to simulate both mechanisms of ACF morphogenesis using different locations for the first abnormal cell. Inside crypts the abnormal cell density is characterized by a convection-diffusion equation where the convective velocity obeys to a Darcy's Law [3] and in the intercryptal region is characterized by a diffusive equation. We use a levelset equation in order to track the abnormal region. A similar study has been performed in [2], but only inside the crypt and with a different flux expression that defined the level-set velocity.

The paper is organized as follows. We present in Section 2 the differential coupled convective-diffusive and level-set model, the solution of such model is approximated numerically using a Finite Elements discretized model that is discussed in Section 3. In Section 4 we discuss and show instead some numerical simulations obtained with two different initial configurations of a single abnormal cell set. Finally in the last section, we state our conclusions and indicate possible future works in this area.

\section{Model}

Each colonic crypt can be represented by a three-dimensional (3-D) cylinder closed at the bottom and with a round opening orifice at the top, see left picture in Figure 1. However, following the approach used in [2], it can also be represented in two-dimensions (2-D) by a circle.

In Figure 1(right) each circle represents a transversal section of the crypt. In this representation the circumference with the largest radius $R$ locates the cells that reside at the top of the crypt (in the orifice), while those at the bottom of the crypt are represented by the unique center of the concentric circumferences.
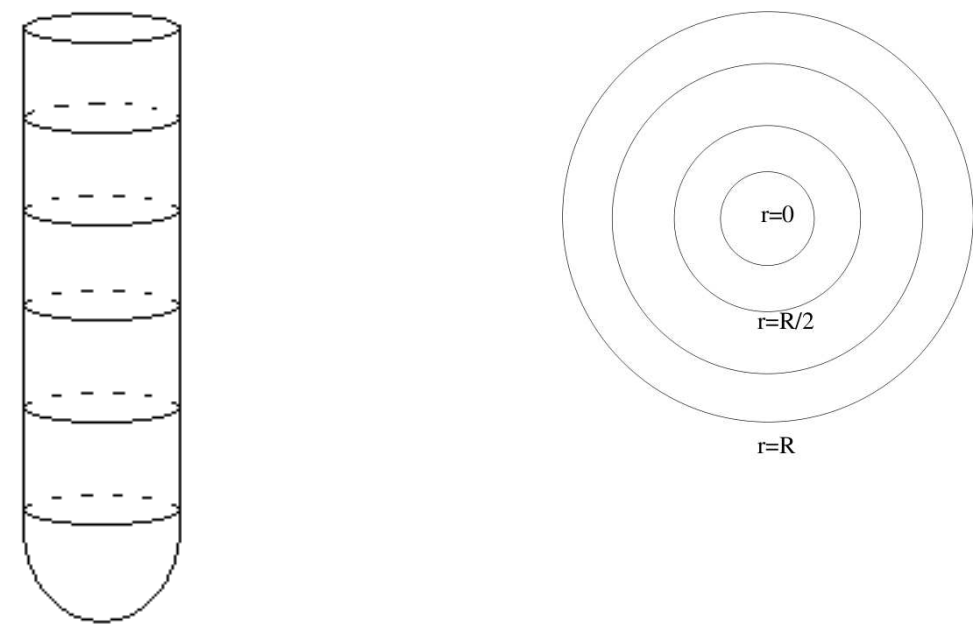

Figure 1. Representation of a single crypt in 3D (left picture) and in 2D (right picture). In the right picture the circumference with radius $r=R$ represents points in the orifice (top) of the crypt.

In general a piece of colon is defined as a rectangular domain that consists of millions of periodic small rectangles with a circle(crypt) at the center and a intercryptal region out- 
side the circle. In this work we will consider only two of these small rectangles which are represented in Figure 2. Here each circle represents the projection of a crypt in the plan, as described at the beginning of this section, thus circles in Figure 1 are in $\Omega_{i n}$, and the rest of the domain, that is the intercryptal region, is $\Omega_{e x t}$.

L1

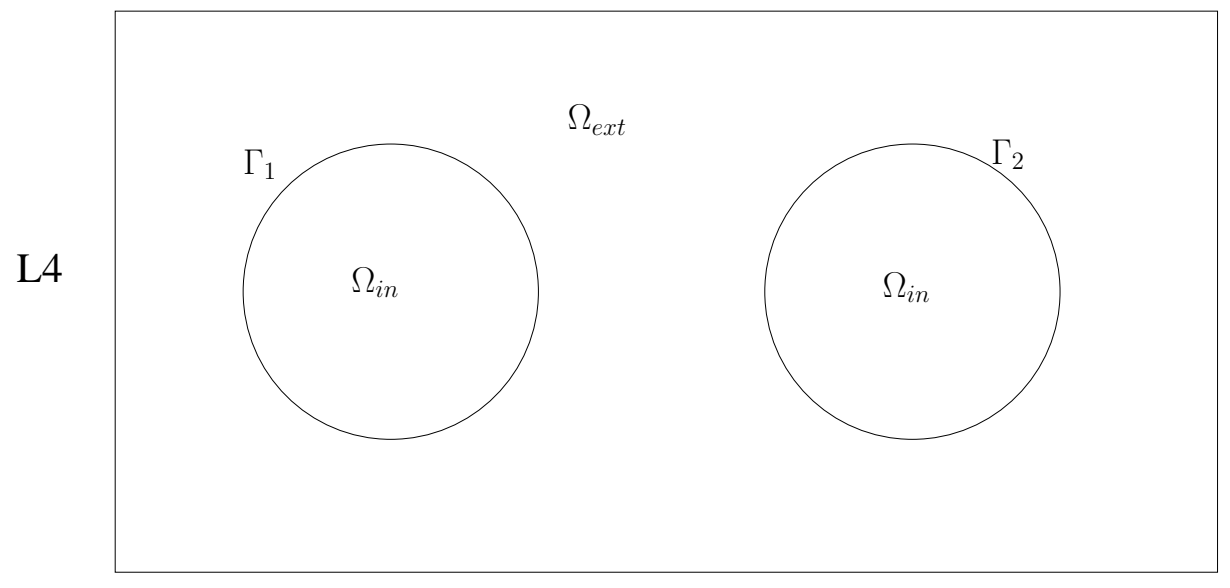

L3

Figure 2. Geometrical domain used in the numerical simulations.

In the crypt region $\Omega_{i n}$, we use a convective-diffusive model similar to that used in our previous model, see [3]. The main difference with respect that model, is that now we consider the dynamics of normal and abnormal cells inside as well as outside the crypts. Their density at time $t$ and in position $(x, y)$ in the entire domain $\Omega:=\Omega_{\text {in }} \cup \Omega_{\text {ext }}$ is denoted by $N=N(x, y, t)$ and $C=C(x, y, t)$ for the normal and the abnormal cells, respectively. Though abnormal cells have larger volume with respect to normal cells, see [4], we assume that all cells have the same volume. Thus [ the ] overall density hypothesis in $\Omega$ is

$$
N+C=1 \text {. }
$$

This allows us to simplify the model, reducing the number of parameters, and enabling the possibility to extend it to more realistic situations in future works, read the discussion in Section 6.

Moreover, we suppose that both normal and abnormal cells move with the same convective velocity in $\Omega_{\text {in }}$ defined by a Darcy's Law

$$
v=-\nabla p .
$$

The model described yields the following differential system in $\Omega_{i n}$ :

$$
\begin{cases}\frac{\partial N}{\partial t}-\nabla \cdot(\nabla p N)=\nabla \cdot\left(D_{1} \nabla N\right)+\alpha_{1} N & \text { in } \Omega_{\text {in }} \times(0, T), \\ \frac{\partial C}{\partial t}-\nabla \cdot(\nabla p C)=\nabla \cdot\left(D_{2} \nabla C\right)+\alpha_{2} C & \text { in } \Omega_{\text {in }} \times(0, T),\end{cases}
$$

where $D_{1}, \alpha_{1}$ and $D_{2}, \alpha_{2}$ are respectively the diffusion coefficient and the rate of birth of normal and abnormal cells in $\Omega_{i n}$. 
If we sum the two equations in (3) and use the relation (2), the following differential system in the unknowns $C$ and $p$ is obtained:

$$
\begin{cases}\frac{\partial C}{\partial t}-\nabla \cdot(\nabla p C)=\nabla \cdot\left(D_{2} \nabla C\right)+\alpha_{2} C & \text { in } \Omega_{\text {in }} \times(0, T) \\ -\Delta p=\nabla \cdot\left(\left(D_{2}-D_{1}\right) \nabla C\right)+\left(\alpha_{2}-\alpha_{1}\right) C+\alpha_{1} & \text { in } \Omega_{\text {in }} \times(0, T) .\end{cases}
$$

Since in the inter-cryptal region $\Omega_{\text {ext }}$ only the abnormal cells can spread and proliferate [9], we assume that here the density $C(t)$ must verify the diffusion equation

$$
\frac{\partial C}{\partial t}=\nabla \cdot\left(D_{2}^{*} \nabla C\right)+\alpha_{2}^{*} C \quad \text { in } \Omega_{e x t} \times(0, T) .
$$

As the proliferative activity of normal and abnormal cells is higher at the bottom of the crypt with a slow decreasing along the crypt axis [3, 4], we will consider rates of birth, $\alpha_{1}$ and $\alpha_{2}$, that are decreasing functions with respect the distance from the center of the circles, see in Section 5 the equations (15)-(16).

The proliferative activity in colonic crypts can promote mutations of normal into abnormal cells. In the inter-cryptal region the normal cells are in a apoptotic phase, so the abnormal cells, are expected to diffuse less than inside the crypts. Therefore we will assume that $D_{2}^{*}<D_{2}$. Finally we mention that it is theoretically known, read the discussion in [9], that abnormal cells proliferate more than normal cells in $\Omega_{i n}$. This leads us to use a proliferative rate $\alpha_{2}$ (for abnormal cells) in $\Omega_{\text {in }}$ that is larger with respect to that used for normal cells $\left(\alpha_{2}>\alpha_{1}\right)$, see their expression in equations (15)-(16) in Section 5.

\subsection{Boundary conditions}

In order to solve the differential systems (4) and (5), we need to impose some boundary conditions in the crypt interface boundaries $\Gamma_{1}, \Gamma_{2}$ (see Figure 2) and also in the edges $L_{i}, i=$ $1, \ldots, 4$ of the boundary of $\Omega$. We use the following boundary transmission conditions

$$
\begin{aligned}
{[|C|] } & =0 & & \text { on } \Gamma_{i} \times(0, T), \\
\frac{\partial p}{\partial n} C+D_{2} \frac{\partial C}{\partial n} & =D_{2}^{*} \frac{\partial C}{\partial n} & & \text { on } \Gamma_{i} \times(0, T) .
\end{aligned}
$$

The first condition guarantees that the density is continuous through the interface boundaries $\Gamma_{i}$, while the second condition asserts, using the equations $(4)_{1}$ and (5), that the normal flux in $\Gamma_{i}$ is continuous in the interface boundaries. We impose also an homogeneous Dirichlet and a Neumann boundary condition in the lateral boundaries $L_{2}, L_{4}$

$$
\begin{array}{rlr}
\frac{\partial C}{\partial n} & =0 & \text { on }\left[L_{2} \cup L_{4}\right] \times(0, T), \\
C(x, y, t)_{L_{2}} & =C(x, y, t)_{L_{4}}=0, &
\end{array}
$$

and a Neumann-periodic condition in $L_{1}, L_{3}$

$$
\begin{array}{rlr}
\frac{\partial C}{\partial n} & =0 & \text { on }\left[L_{1} \cup L_{3}\right] \times(0, T) \\
C(x, y, t)_{L_{1}} & =C(x, y, t)_{L_{3} .} &
\end{array}
$$


These conditions in $L_{i}$, with $i=1,3$, are used to model the colon in a hypothetical situation in which a cut is applied in $L_{1}$ and then the unfolded colon is rolled in the plane, read the discussion in Section 6.

We impose also the following initial condition for the density $C$ :

$$
C(x, y, 0)=C_{0}(x, y) \quad \text { in } \Omega
$$

where $C_{0}(x, y)$ is a given function representing the initial density distribution of abnormal cells.

\subsection{Level Set tracking}

In order to predict the evolution of abnormal cells, we need to model not only their density, but also the dynamics of the sets containing such cells. The boundaries of these abnormal sets can in fact mark colonic areas where cells are in a configuration that can bring to the formation of an ACF.

We use a level set function $\Phi(x, y, t)$ at level 0 to track in space and time the boundary

$$
B(t)=\{(x, y) \in \Omega: \Phi(x, y, t)=0\} .
$$

of an abnormal cell set with interior part denoted by $D(t)$,

$$
D(t)=\{(x, y) \in \Omega: \Phi(x, y, t) \leq 0\} .
$$

Given the normal velocity $v_{\Phi, \mathbf{n}}:=\mathbf{v}_{\Phi} \cdot \mathbf{n}$ of the level set, see [5], we have that the level set function satisfies

$$
\frac{\partial \Phi}{\partial t}+v_{\Phi, \mathbf{n}}|\nabla \Phi|=0
$$

where $\mathbf{v}_{\boldsymbol{\Phi}}$ is the velocity and $\mathbf{n}$ is the outward normal of the level set considered. This velocity is equal to the ratio between the flux, obtained using equations $(4)_{1},(5)$, and the cell density $C(x, y, t)$ of the abnormal cells

$$
\mathbf{v}_{\boldsymbol{\Phi}}(x, y, t)= \begin{cases}-\left(\nabla p+\frac{D_{2} \nabla C}{C}\right) & \text { in } \Omega_{i n} \times(0, T) \\ -\frac{D_{2}^{*} \nabla C}{C} & \text { in } \Omega_{e x t} \times(0, T)\end{cases}
$$

\section{Numerical model}

In this section we use a finite element discretization (with a semidiscrete Galerkin formulation) applied to the model systems (4) and (5). Multiplying equations (4) $)_{1}$ and (5) by a test function $\varphi$ and integrating by parts we obtain

$$
\int_{\Omega} \frac{\partial C}{\partial t} \varphi d x d y+\int_{\Omega_{i n}}\left(C \nabla p+D_{2} \nabla C\right) \nabla \varphi d x d y+\int_{\Omega_{e x t}}\left(D_{2}^{*} \nabla C\right) \nabla \varphi d x d y=\int_{\Omega} \alpha_{2} C \varphi d x d y .
$$


Applying on the other hand the semidiscrete Galerkin formulation to the pressure equation $(4)_{2}$, we obtain

$$
\int_{\Omega_{i n}} \nabla p \nabla \varphi d x d y=-\int_{\Omega_{i n}}\left(\left(D_{2}-D_{1}\right) \nabla C\right) \nabla \varphi d x d y+\int_{\Omega_{i n}}\left(\alpha_{2}-\alpha_{1}\right) C \varphi d x d y+\int_{\Omega_{i n}} \alpha_{1} \varphi d x d y .
$$

The equations (10)-(11) result finally in the following discrete system

$$
\begin{cases}M \frac{\partial C}{\partial t}+\left(E_{\nabla p}+K\left(D_{2}, D_{2}^{*}\right)\right) C=M\left(\alpha_{2}\right) C & \text { in } \quad \Omega \times(0, T), \\ K_{\Omega_{i n}} p=-\left(K_{\Omega_{i n}}\left(D_{2}-D_{1}\right)-M_{\Omega_{i n}}\left(\alpha_{2}-\alpha_{1}\right)\right) C+M_{\Omega_{i n}} \alpha_{1}(\cdot) & \text { in } \quad \Omega_{\text {in }} \times(0, T),\end{cases}
$$

where $\alpha_{1}(\cdot)$ is the vector with the value of $\alpha_{1}$ in the $\Omega_{\text {in }}$ nodes. We have represented in (12) by $K$ and $M$ the stiffness and the mass matrix, respectively, and used an obvious notation for these matrices in $\Omega_{i n}, \Omega_{\text {ext }}$ and $\Omega$. The matrix $K\left(D_{2}, D_{2}^{*}\right)$ is instead the stiffness matrix in $\Omega$ that it is equal to $K_{\Omega_{i n}}\left(D_{2}\right)$ in $\Omega_{i n}$ and to $K_{\Omega_{e x t}}\left(D_{2}^{*}\right)$ in $\Omega_{\text {ext }}$.

The matrix $E_{\nabla p}$ has a dimension equal to the number of degrees of freedom $N_{i n}$, used in $\Omega_{\text {in }}$, its coefficients are

$$
\left(E_{\nabla p}\right)_{i, j}=\sum_{k=1}^{N_{i n}} p_{k} \int_{\Omega_{i n}} \varphi_{j} \nabla \varphi_{i} \nabla \varphi_{k} d x, \quad i, j=1, \ldots N_{i n} .
$$

In order to integrate in time the parabolic equation (12) $)_{1}$, we use the following secondorder implicit trapezoidal scheme (Crank-Nicolson method):

$$
M C^{m+1}=M C^{m}-\frac{\delta t}{2}\left(E_{\nabla p}+K\left(D_{2}, D_{2}^{*}\right)-M\left(\alpha_{2}\right)\right)\left(C^{m}+C^{m+1}\right)
$$

where $C^{m}$ is an approximation of $C$ at time $t_{m}$. This equation can be reduced to

$$
\left(M+\frac{\delta t}{2} G\right) C^{m+1}=\left(M-\frac{\delta t}{2} G\right) C^{m}
$$

where we posed $G:=E_{\nabla p}+K\left(D_{2}, D_{2}^{*}\right)-M\left(\alpha_{2}\right)$.

\section{Multi-scale Algorithm}

Two different scales in space and time, respectively, are used to solve the level set equation (8) and the pressure-density system (12). Regarding the time-scaling, we use the time-step $d t$ for solving (8) and $\delta t$ in (14). In particular, we require that the level-set time step $d t$ satisfies the Courant-Friedrich-Levy (CFL) condition, $d t<\left(\frac{\min (d x, d y)}{\max \left|v_{\Phi, \mathbf{n}}\right|}\right)$, where $d x, d y$ are the 2-D space steps used in (8), see Step 3 of the algorithm below. In contrast the time step $\delta t$ is computed implementing an adaptive control step in the integration time interval (for the level-set equation) $[t, t+d t]$, using a classical approach (as that described in [6], Chapter 12). This means that $\delta t$ is always smaller or at most equal to $d t$.

In order to simulate the dynamics of a set of abnormal cells in $[0, T]$ with initial density $C_{0}(x, y)$ and level set function $\Phi^{0}:=\Phi(x, y, 0)$, the following algorithm is implemented: 
- Compute the initial pressure $p(x, y, 0)$ in $\Omega_{i n}$ using $(12)_{2}$ with $C_{0}$ in place of $C$. Let $t^{0}=0$ and $n=0$

- Compute the velocity $\mathbf{v}_{\boldsymbol{\Phi}}$ using (9) in $B\left(t^{0}\right)$, elsewhere $\mathbf{v}_{\boldsymbol{\Phi}}$ is null

- Define the level set time-step $d t=0.5\left(\frac{\min (d x, d y)}{\max \left|v_{\Phi, \mathbf{n}}\right|}\right)$

- While $t^{n}+d t<T$ do

1. Determine the level-set $\Phi$ at time $t^{n+1}:=t^{n}+d t$ integrating the equation (8) forward in time with the following second order Total Variation Diminishing Runge-Kutta scheme:

- First, an Euler step is taken to approximate the solution at $t^{n+1}$

$$
\frac{\widetilde{\Phi}^{n+1}-\Phi^{n}}{d t}+v_{\Phi^{n}, \mathbf{n}}\left|\nabla \Phi^{n}\right|=0
$$

here $\Phi^{n}$ is the current approximation of the level set function $\Phi$ at time $t^{n}$,

- Then a second Euler step is used to advance the solution at $t^{n+1}+d t$,

$$
\frac{\widetilde{\Phi}^{n+2}-\widetilde{\Phi}^{n+1}}{d t}+v_{\widetilde{\Phi}^{n+1}, \mathbf{n}}\left|\nabla \widetilde{\Phi}^{n+1}\right|=0 .
$$

The velocity $v_{\widetilde{\Phi}^{n+1}, \mathbf{n}}$ used above is computed using the following strategy:

* Compute the density $C$ and the pressure $p$ both at time $t^{n+1}$ in the set $A:=\left\{(x, y) \mid \widetilde{\Phi}^{n+1}<0\right\}$ using the same approaches presented in steps 2 and 3 below,

* Compute $v_{\widetilde{\Phi}^{n+1}}$ (using (9)) and afterwards $v_{\widetilde{\Phi}^{n+1}, \mathbf{n}}$ in the set $A$, elsewhere pose them equal to zero.

- Average the approximations at time $t^{n}$ and $t^{n+1}+d t$ to obtain the approximate solution at time $t^{n+1}$

$$
\Phi^{n+1}=\frac{1}{2} \Phi^{n}+\frac{1}{2} \widetilde{\Phi}^{n+2} .
$$

The gradients $\nabla \Phi$ are computed using a second order Hamilton-Jacobi ENO upwind method, that is a second order accurate polynomial interpolation (see [5], Chapter 3).

Define $B\left(t^{n+1}\right):=\left\{(x, y) \mid \Phi^{n+1}<0\right\}$ and apply Step 2 and Step 3 in $B\left(t^{n+1}\right)-A$

2. Compute the density $C$ at time $t^{n+1}$ solving the linear system (14) in $\left[t^{n}, t^{n+1}\right]$ using the adaptive control step described previously

3. Compute the pressure $p$ at time $t^{n+1}$ solving the pressure equation (12) using the density $C$ computed in the previous step

4. Determine the velocity $\mathbf{v}_{\boldsymbol{\Phi}}$ in $\Omega_{\text {in }}$ and $\Omega_{e x t}$ in $B\left(t^{n+1}\right)$ using (9) elsewhere we pose $\mathbf{v}_{\boldsymbol{\Phi}}=0$

5. Update the index $n: \quad n=n+1$ 
6. Define the new time-step for the level-set equation: $d t=0.5\left(\frac{\min (d x, d y)}{\max \left|v_{\Phi, \mathbf{n}}\right|}\right)$ end While.

Note that steps 2 and 3 of the algorithm are performed only in a computational domain defined by $A$ or $B\left(t^{n+1}\right)-A$. This permit us to avoid to compute density and pressure in all the domain $\Omega$ and to save then computational resources as the memory and the elapsing time needed in the numerical simulations.

\section{Numerical Results}

We use diffusion coefficients that are constant in space and time: $D_{1}=2, D_{2}=$ $1, D_{2}^{*}=1 / 10$. We suppose that the crypt orifice radius $R$ is equal to 1 , and then we use a geometrical domain that has dimensions scaled based on such assumption. Let $r(x, y)$ be the distance between a point $(x, y)$ in a crypt and the center of such crypt. Taking into account that for normal colonic crypts we only have proliferative activity until two thirds of the height, we define the proliferative rates $\alpha_{1}$ and $\alpha_{2}$ in the following way

$$
\begin{gathered}
\alpha_{1}(x, y)=\left\{\begin{array}{cl}
(r(x, y)-2 / 3)^{2} & \text { if } r(x, y)<2 / 3 \\
0 & \text { if } r(x, y) \geq 2 / 3
\end{array}\right. \\
\alpha_{2}(x, y)=\left\{\begin{array}{cl}
(2 / 9) \cdot\left((r(x, y)-1)^{2}+1\right) & \text { if } r(x, y)<1 \\
2 / 9 & \text { if } r(x, y) \geq 1
\end{array} .\right.
\end{gathered}
$$

Note that $\alpha_{2}$ in (16) is always positive and continuous in the boundary of each crypt. This allows us to have abnormal cells that proliferate inside as well as outside the crypts. Note also that since the normal cells cannot be renewed outside the crypts (because they are in a apoptosis phase), we have $\alpha_{1}=0$ in $\Omega_{\text {ext }}$.

In the next two paragraphs we present two initial configurations of the problem: the first represents a set of abnormal cells in the inter-cryptal region and the second has a set of abnormal cells in the center of a crypt. The figures 3 and 4 show some snapshots of the simulation of the boundary cell set (in black) and density (in colour) performed in Matlab. These are displayed for particular simulation times that are useful and relevant in the discussion of the numerical results.

\subsection{Initial abnormal cells in $\Omega_{e x t}$ and discussion}

We describe here the numerical simulation results obtained during the evolution of an abnormal cell set with uniform density $C=1$, initially located and centered in $\Omega_{\text {ext }}$ between the two crypts. The left picture depicted at the top of Figure 3 displays the initial configuration of the cell set.

The simulation shows that the abnormal cells diffuse rapidly in $\Omega_{e x t}$ in the time interval $[0,0.5]$, but the closer these cells are to the crypt boundary, the less they propagate.

At time $t=0.5$ the abnormal cells start to enter in the crypt. We observe in fact that the level set, that marks the boundary of the cell set, overlaps the crypt boundaries $\Gamma_{i}$, see the right picture depicted in the middle of Figure 3. 

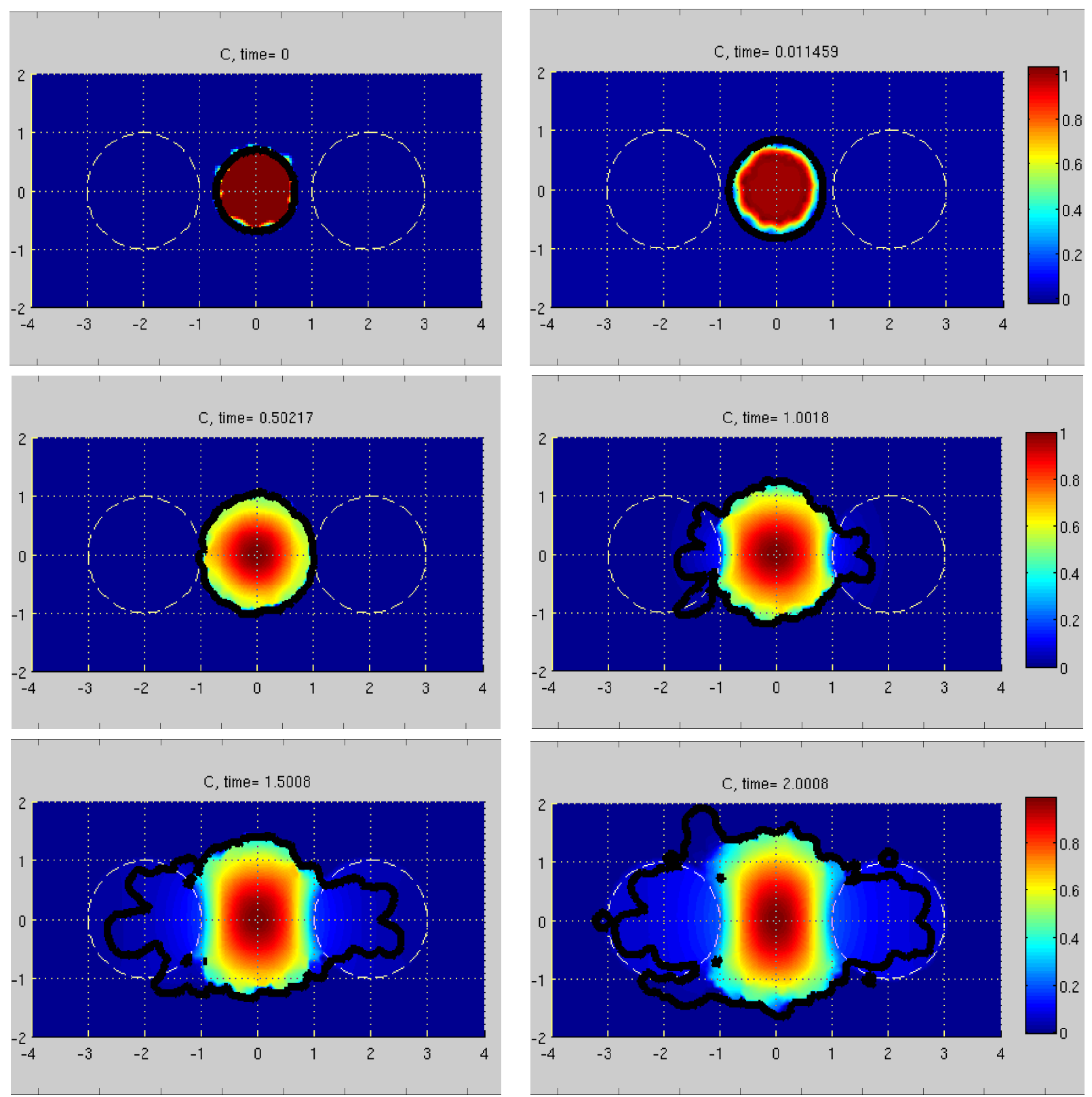

Figure 3. Density and level-set (in black) at time 0, 0.01 (in the top pictures) $0.5,1$ (in the middle pictures), 1.5, 2 (in the bottom pictures). Dashed lines mark the two crypt orifices.

When time moves forward the cells spread inside the crypts and, at time $t=2$, the level set reaches the boundary of the domain $\Omega$, see the right picture depicted at the bottom of Figure 3. Note that at time $t=2$ in spite of abnormal cells are filling both crypts with a low density, most of them remain in the inter-cryptal region and diffuse only vertically avoiding to enter in such crypts. This phenomenon is due to the pressure exerted by the cells that opposes the uniform diffusion in each direction that is expected in $\Omega_{\text {ext }}$.

\subsection{Initial abnormal cells in $\Omega_{i n}$ and discussion}

In this section, we describe the evolution of abnormal cells when they are initially located at the bottom of a crypt with the uniform density $C=1$. This initial configuration is 
displayed in the left picture at the top of Figure 4.

This figure shows that cells diffuse more rapidly inside the crypts than outside. In fact, the cells reach now the orifice of the crypt at time 0.002, while in the previous case, see Figure 3 , this was observed in $\Omega_{\text {ext }}$ at time $t=0.5$. Moreover at time $t=0.01$ we notice that some cells have already overpassed the orifice because they are located in $\Omega_{e x t}$, see the left picture depicted in the middle of Figure 4. The rapid propagation of cells observed in the crypts is due
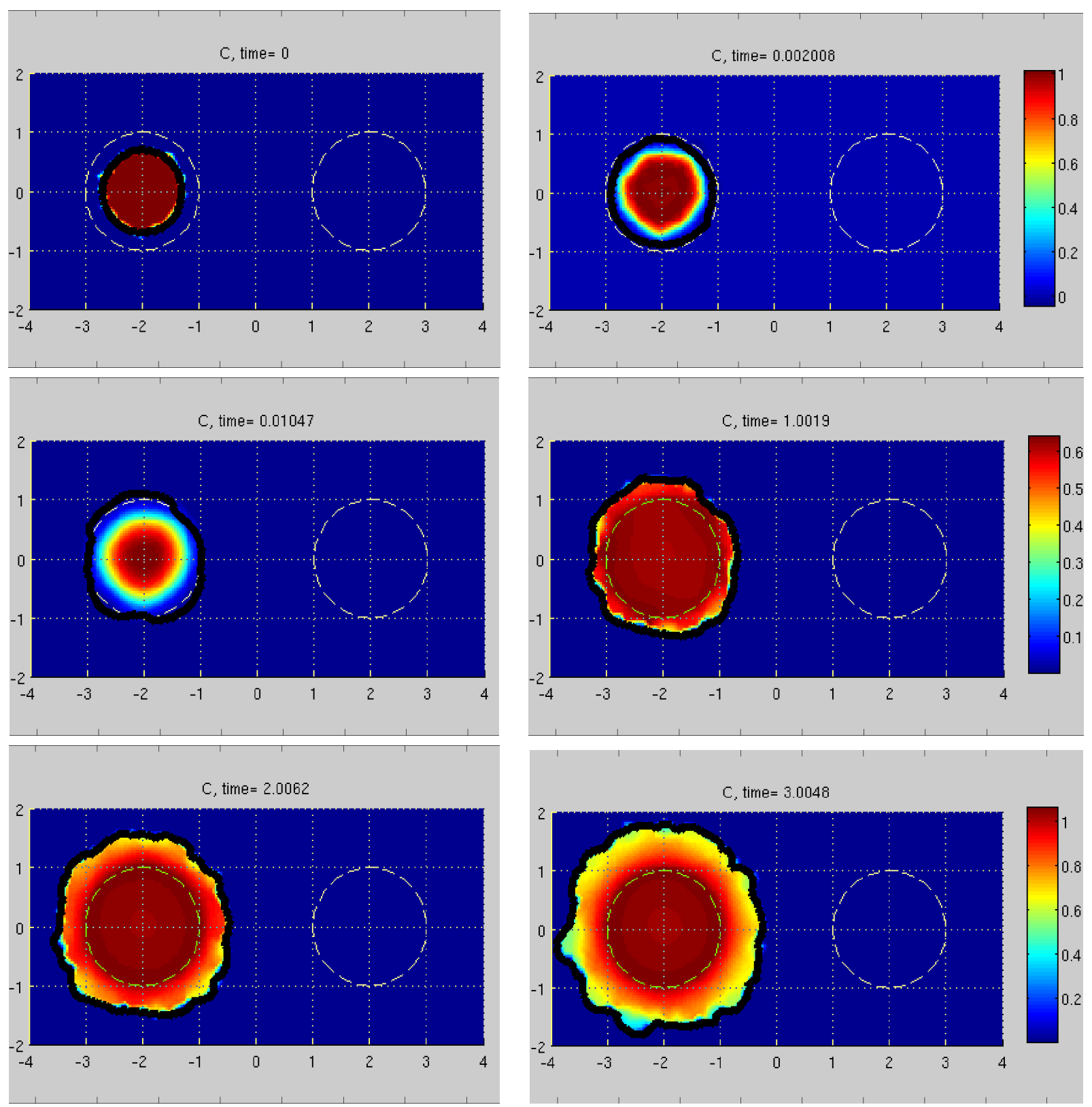

Figure 4. Density and level-set at time 0, 0.002 (in the top pictures) $0.01,1$ (in the middle pictures) and 2, 3 (in the bottom pictures).

by the presence of a convective velocity in (4), that is instead supposed null in $\Omega_{\text {ext }}$ because no pressure is exerted there. This loss of pressure in $\Omega_{e x t}$ explains also the slow spread of abnormal cells, when they exit from the crypts, with respect to the propagation observed before in $\Omega_{i n}$, see bottom pictures in Figure 4. We note finally that, when the boundary 
of the cell set has overpassed the crypt boundaries, the density starts to increase more in a neighborhood of the boundaries $\Gamma_{i}$ than inside the crypts. These dynamics are explained by the fact that abnormal cells that leave the crypts cannot re-enter in $\Omega_{i n}$ due to the pressure exerted by the cells that are exiting from $\Omega_{i n}$.

\section{Conclusion and Future work}

We have presented a model for tracking density and pressure of abnormal cells inside the colon. We used a coupled convection-diffusion level set model that is able to reproduce some dynamical aspects of abnormal cells in colonic crypts and track also the abnormal cell set boundary.

Based on the results presented in Section 5.1, we infer that with this model we have represented the bottom-up and the top-down theories described in the Introduction. Regarding the top-down theory, we observe that our parameter choice permits the propagation from the inter-cryptal region to the adjacent crypts of a limited number of abnormal cells, see for instance the low density depicted in Figure 3. A different parameters' choice can be used to increase the density of such cells. We think, for example, that a possible modification of the Darcy's law, such as $v=\mu \nabla p$ with $\mu$ smaller than 1 will reduce the pressure exerted in $\Omega_{\text {in }}$ and this could permit the entrance of more abnormal cells inside the crypts.

In the numerical simulations a domain with only two crypts was considered. However, a reasonable model should consider more than just two crypts, this will be studied in a future research. We think also to consider, in a future work, several abnormal cell sets that are able to merge and to simulate then the dynamics of their boundaries and densities.

In the future, we intend also to associate an height function to the density and then to simulate the evolution of abnormal cells in 3-D. In this way we could model and show that the formation of ACF is a consequence of the accumulation of abnormal cells. We may describe in fact a growth in the third dimension of the abnormal cell set in the colon epithelium instead of just describing an increase of the density in two-dimensions as done in this work.

\section{Acknowledgements}

This work was partially supported by Contract UTAustin/MAT/0009/2008 from the UT Austin | Portugal Program (http://www.utaustinportugal.org/) and by the Centre for Mathematics of the University of Coimbra and Fundao para a Ciłncia e a Tecnologia, through the European program COMPETE/FEDER. 


\section{REFERENCES}

[1] Boyle, P. and LevinSteward, B.,"World Cancer Report",IARC Press, Lyon, France 2008.

[2] Figueiredo I.N., Leal C., Leonori T., Romanazzi G., Figueiredo P.N., Donato M.M., "A coupled convection-diffusion level set model for tracking epithelial cells in colonic crypts”. Procedia Computer Science 1, 955-963, 2010.

[3] Figueiredo I.N., Leal C., Romanazzi G., Engquist B., Figueiredo P., "A convectiondiffusion-shape model for aberrant colonic crypt morphogenesis". Computing and Visualization in Science 14(4), 157-166, 2011.

[4] Holt P. R. et al., "Modulation of Abnormal Colonic Epithelial Cell Proliferation and Differentiation by Low-Fat Dairy Foods". Journal of American Medical Association 280(12), 1074-1079, 1998.

[5] Osher S., Fedkiw R., "Level set methods and dynamic implicit surfaces". Applied Mathematical Sciences 153, Springer-Verlag, New York, 2003.

[6] Pina H., "Métodos numéricos". McGraw-Hill, Lisboa, 1995.

[7] Preston S. L. et al., "Bottom-up Histogenesis of Colorectal Adenomas: Origin in the Monocryptal Adenoma and Initial Expansion by Crypt Fission”. Cancer Research 63, 3819-3825, 2003.

[8] Shih I. M. et al.,"Top-down morphogenesis of colorectal tumors". Proceedings of the National Academy of Sciences of the United States 98(5), 2640-2645, 2001.

[9] van Leeuwen I. M. M., Byrne H. M., Jensen O. E., King J. R., "Crypt dynamics and colorectal cancer: advances in mathematical modelling", Cell Proliferation 39, 157-181, 2006. 